\title{
Mundo de la vida, comprensión y acción intersubjetiva en la sociología fenomenológica de Alfred Schütz
}

\section{World of life, comprehension and intersub- jective action in Alfred Schuitz's phenomeno- logical sociology}

Héctor Mora Nawrath ${ }^{1}$

\begin{abstract}
RESUMEN
El artículo elabora una aproximación descriptiva y analítica respecto de tres conceptos centrales en la propuesta de Alfred Schütz: 'mundo de la vida' (Lebenswelt), 'comprensión' (Verstehen) y 'acción intersubjetiva'. Explora el sentido del 'mundo de la vida' como realidad eminente, así como la multiformidad que éste adquiere desde la perspectiva del actor social; revisa el concepto de 'comprensión' en tanto capacidad del actor, como en su relación con la conformación del mundo social; y reflexiona respecto a las características que adquiere la acción social subjetiva e intersubjetiva, situándola en los marcos espaciotemporales.
\end{abstract}

Palabras clave: Mundo de la vida, comprensión, acción social, intersubjetividad.

\section{ABSTRACT}

This article develops a descriptive and analytical approach to three central concepts in Alfred Schütz's proposal: 'World of life' (Lebenswelt), 'comprehension' (Verstehen) and 'intersubjective action'. It explores the meaning of the 'world of life' as an eminent reality, and its multiform nature from the perspective of the social actor; it reviews the concept of 'comprehension' as the actor's capacity and how this comprehension is related with the conformation of the social world; and it reflects on the characteristics acquired by subjective and intersubjective social action, placing them in a space-time framework.

Key Words: World of life, comprehension, social action, intersubjectivity.

Magíster en Ciencias Sociales y Doctorando en Ciencias Sociales Universidad Nacional de La Plata, Argentina. Docente de la Escuela de Antropología de la Universidad Católica de Temuco y becario de posgrado del Consejo Nacional de Ciencia y Tecnología, Gobierno de Chile. hectmora@uct.cl 


\section{Introducción}

Sin lugar a duda, uno de los mayores aportes de Schütz radica en trazar un nexo entre la filosofía fenomenológica y la teoría social (López 1995; Reynoso 1998)², llevando a cabo un programa que contribuye al desarrollo de diversas corrientes inscritas en la 'sociología comprensiva' o 'sociología fenomenológica’ (Mèlich 1932[1993]), como también a marcos interpretativos de otras disciplinas de las Ciencias Sociales (Reynoso 1998). Schütz organiza de manera creativa la contribución de autores como Husserl, Bergson, Scheler, James y Weber, entre otros, y desarrolla una aproximación teórica dirigida a comprender la estructura y distribución social del sentido común (López 1995) o como señala Alexander (1987 [1992]), una fenomenología mundana. Schütz "inserta la actividad intencional trascendental en el contexto de órdenes culturales particulares" (Alexander 1987[1992]: 203), poniendo énfasis en como, desde la 'actitud natural', se hace posible la constitución y reproducción del mundo social, y el papel fundamental que en ello juega la dimensión subjetiva y las relaciones intersubjetivas que establecen los diversos actores sociales (Salas 2006). Schütz abandona la pretensión de profundizar en la fenomenología trascendental y sitúa su programa en la 'fenomenología de la actitud natural' como comprensión de la realidad eminente de la vida de sentido común (Natanson 1932 [2003]), convencido que desde esta era posible encontrar claves que permitieran la fundamentación de una sociología comprensiva en tanto ciencia que “... produce construcciones de segundo grado derivadas de las que tienen lugar en la vida cotidiana" (López, 1995:56).

Este trabajo busca relevar algunos aspectos fundamentales de la propuesta de Schütz, particularmente mundo de la vida, comprensión y acción social e intersubjetividad. Para ello, la propuesta se estructura en tres apartados a partir de los cuales se buscará profundizar en los tópicos antes señalados.
El primer apartado, denominado El mundo de la vida cotidiana como 'realidad eminente', presenta las ideas centrales de Schütz acerca de la definición de 'mundo de la vida' (Lebenswelt), haciendo hincapié en su fundamentación como 'realidad eminente' y su relación básica con las nociones de 'sentido de realidad', 'actitud natural' y 'acción'. El segundo apartado, 'La comprensión (Verstehen): conformación del mundo social y subjetividad', busca describir las características y el papel que cumple la noción de Verstehen como capacidad de los actores sociales en la conformación del mundo social, enfatizando el elemento subjetivo y la relación de tal noción con el concepto de 'realidad social'. El tercer apartado (subjetividad, intersubjetividad y temporalidad en la acción social) describe el papel de la subjetividad e intersubjetividad en la acción como manifestación del actor en la vida cotidiana, señalando su relación con la 'conciencia', la 'atención a la vida' y el 'estado de alerta', incorporando la dimensión temporal a través de conceptos como 'proyecto', 'sentido', 'presente vívido'.

\section{El mundo de la vida cotidiana como reali- dad eminente}

"Únicamente en el mundo de la vida puede constituirse un mundo circundante, común y comunicativo. El mundo de la vida cotidiana es, por consiguiente, la realidad fundamental y eminente del hombre" (Schütz y Luckmann 1973 [1977]:25).

\subsection{El mundo de la vida o Lebenswelt}

Desde un punto de vista analítico, se podría decir que 'mundo de la vida' o Lebenswelt corresponde a una categoría que se piensan como constitutiva de cualquier experiencia precientífica (Luckmann 1992 [1996]), a partir de la cual se ha buscado definir la 'naturaleza de la realidad social'”, esto, como alternativa a la noción que sirve de sustrato a la ciencia experimental y que se buscaba hacer extensible para todas las ciencias -incluyen-

Reynoso señala que Ciacci, Friedrichs y Gouldner estipulan que “... ha sido Schütz quien transfirió una dimensión filosófica interpretativa, fenomenológica, a una sociología atestada de tecnicismo, manipulada por el establishment y necesitada de alternativas humanísticas" (1998:96).

3 Se hace referencia a la forma y naturaleza que adquiere la realidad y, por ende, lo que puede ser conocido. Desde la perspectiva de Schütz debiésemos hablar de ámbitos de sentido, pues como este señala “... lo que constituye la realidad es el sentido de nuestras experiencias, y no la estructura ontológica de los objetos” (1962 [2003]: 215).8 
do- la social. Desarrollada en primera instancia por Husserl, es retematizada por Schütz -retomando este término en sus escritos más tardíos $-{ }^{4}$, centrándose particularmente en la caracterización del 'mundo social de los contemporáneos' (soziale Mitwelt) o 'mundo del presente cotidiano' (Mèlich (1932 [1993]) en tanto dominio clave para la comprensión de la realidad eminente de la vida de sentido común $y$, por ende, de la acción e interacción social (Mèlich 1932 [1996]; Natanson 1962 [2003]).

Según López (1995), la principal diferencia entre el 'mundo de la vida' (Le benswelt) en Husserl y en Schütz, radica en que para el primero este es fruto del análisis regresivo que parte de la ciencia y halla sus fundamentos mediante la reducción mientras para el segundo, es el punto de partida del conocimiento actual y posible, centrándose en el mundo de la actitud natural en tanto vivenciado. Tal como es posible consignar, Lebenswelt en Husserl es un término compuesto por dos vocablos: Leben y Welt. Leben o vida, no es vista en un sentido fisiológico, sino más bien se concibe como algo dinámico, que actúa de acuerdo a ciertos fines, creadora de forma espirituales, de cultura y como la unidad de una historicidad. Welt correspondería al mundo como totalidad, y en tanto existente, es predado universal pasivo que antecede a toda actividad de juicio y es independiente, siendo este el sustrato absoluto en el sentido estricto de la independencia absoluta (Novella 1998, Salas 2006). Lebenswelt “... es, ante todo, en cuanto un núcleo, mundo sensible aparente y caracterizado como 'ahí delante', dado en simples intuiciones de experiencia $y$, eventualmente, captado actualmente... dado como horizonte de todas las inducciones, dotadas de sentido" (Husserl en Milone 2007:90).

Así definido, el 'mundo de la vida' es el ámbito de la formación del sentido (Sinnbildung) y de la significatividad; es el fundamento final (Endstiftung) en la historia y el tiempo (Novella 1998). Es el mundo que se presenta a la percepción, y en el cual encontramos cosas, animales, hombres, y donde el 'yo mismo' es objeto del mundo y sujetos para el mundo; este aparece en los actos de percepción, valoración y decisión como provisto de sentido en tanto cosa material, utensilio, objeto cultural, animal, etc. (Bengoa 1992). En 'actitud natural' todos estos objetos se consideran como siendo 'en-sí' y no tomamos conciencia de que son lo que son en virtud de nuestros actos; la reflexión fenomenológica, como reducción trascendental, hace aparecer la relación entre conciencia de mundo y mundo, mostrando las distinta formas de darse del objeto (Gegebenheitsweisen), las que están condicionadas subjetivamente al depender de nuestra manera de dirigirnos a él. Para Husserl, la percepción no implica el contemplar desinteresado, sino el acto de una conciencia inmersa en el 'mundo de la vida', que toma conocimiento de las cosas en las acciones motivadas por intereses vitales o prácticos. En palabras de Milone (2007), 'mundo de la vida' sería horizonte vital de posibilidad, base universal de la que surgen deseos, intereses y metas humanas, y fundamento necesario de la experiencia para la percepción, juicio, conocimiento y acción. Para Husserl, "Toda cosa que aparece en la percepción es esencialmente una cosa del mundo. Recíprocamente, el mundo es conocido solo como horizonte universal de las cosas realmente conocidas. Por eso, el mundo no tiene el mismo modo de ser de las cosas: las cosas son singulares (elementos de una pluralidad), el mundo es único" (Bengoa 1992:49).

Para Schütz, 'mundo de la vida' se constituye en una categoría central en su propuesta teórica. Así, los múltiples términos que Schütz utiliza en su obra ${ }^{5}$, ellos expresan el mismo sentido (Natanson 1962[2003]; Leal 2006; Salas 2006) ${ }^{6}$; hacen referencia al mundo social intersubjetivo que el actor vivencia en

\footnotetext{
V. gr. El problema de la realidad social (1962 [2003]), Estudios sobre teoría social (1964 [1974]) y Estructura del mundo de la vida (1973 [1977]).

5 V. gr. En el texto “La construcción significativa del mundo social” (1932 [1993]) es común la expresión 'mundo social’; en “El problema de la realidad social” (1962 [2003]) utiliza expresiones como 'realidad social', 'mundo de la vida' y 'mundo intersubjetivo'; en “Estudios sobre teoría social” (1964 [1974]), ‘mundo cotidiano', 'mundo social’; en “Estructura del mundo de la vida" (1973 [1977]), 'mundo de la vida cotidiana'.

6 V. gr. Gibert (1999) profundiza en la diferenciación conceptual entre 'mundo de la vida' y 'mundo cotidiano' en la obra de Schütz, afirmando que el “... 'mundo de la vida' es principalmente objetivo y estático, ahistórico; mientras que el 'mundo cotidiano' es el mundo construido por el hombre y su alter (luego, es un mundo intersubjetivo); dinámico e histórico” (p. 61). Al parecer este autor confunde, por un lado, mundo de la vida o mundo cotidiano con una de sus dimensiones, es decir,
} 
el cotidiano en 'actitud natural'7. Así, podemos hablar del 'mundo de la vida' en un sentido genérico, denotando ciertas características comunes a todos los 'mundos' o 'realidades' contenidas o posibles. Una de estas características refiere al reconocimiento de que el 'mundo de la vida' es un mundo predado en tanto creación de nuestros antecesores, aun cuando en la interacción me es posible modificarlo. Así Schütz señala que

\begin{abstract}
"Nací, por así decirlo, en este mundo social organizado, y crecí en él. Mediante el aprendizaje y la educación, mediante experiencias y experimentos de todo tipo, adquiero cierto conocimiento mal definido de este mundo y sus instituciones. Los objetos de ese mundo me interesan, sobre todo, en la medida en que determinan mi propia orientación, en que promueven o traban la realización de mis propios planes, en que constituyen un elemento de mi situación que debo aceptar o modificar, en la medida en que son la fuente de mi felicidad o intranquilidad (1967 [1974]:22).
\end{abstract}

Además, el 'mundo de la vida' es intersubjetivo y público (común, accesible a todos y que se hace en la comunicación), e incluye la "... suma total de objetos y sucesos dentro del mundo sociocultural, tal y como los experimenta el sentido común de los hombres que viven su existencia cotidiana entre sus semejantes, con quienes los vinculan múltiples relaciones de interacción" (Schütz 1962 [2003]:74). Es un 'mundo significativo' (die sinnhafte Welt), habitado por semejantes, y por tanto lleva implícito el 'yo' y a los 'otro' como los generadores de la 'cosa significativa'. Al ser una configuración de sentido, el mundo no es objetivo e independiente de la conciencia del individuo (como lo es para el realismo); se manifiesta como una entidad compleja en la cual tienen lugar subuniversos en tanto 'ámbitos finitos de sentido' definidos en un tiempo y espacio (sujeto a la finitud de la existencia o temporalidad vital). Como señala Schütz (1932 [1993]), no es un mundo homogéneo sino que muestra una estructura multiforme, donde cada esferas o regiones es a la vez una manera de percibir y de comprender las vivencias del otro. En definitiva, es el horizonte que comprende la totalidad de las maneras posibles de experienciar (donde ocurre la realización del hombre en su condición humana) y de constituir la vida social, más allá de la experiencia vital de un actor concreto; es decir, es tan amplio como para incluir "todas las modificaciones de actitudes y estados de alerta" (Schütz y Luckmann 1973[1977]:41).

Por otro lado, el 'mundo de la vida' se me presenta siempre como no problemático, y lo vivencio en 'actitud natural', es decir, a la manera de lo típico (de acuerdo a tipificaciones y recetas), dando por sentado lo que acontece sin cuestionamientos hasta 'nuevo aviso', es decir, hasta que tengan lugar situaciones problemáticas. Como señala Schütz,

"En la vida cotidiana, al hombre no le interesa más que parcialmente -y nos atreveríamos a decir que solo excepcionalmente- la claridad de su conocimiento, o sea, la plena percepción de las relaciones entre los elementos de su mundo y los principios generales que gobiernan esas relaciones. Se contenta con disponer de un servicio telefónico en buen funcionamiento $\mathrm{y}$, normalmente, no pregunta en detalle cómo funciona el aparato ni qué leyes de la física hacen posible ese funcionamiento. Compra provisiones en el almacén sin saber cómo se las elabora, y paga con dinero, aunque tiene apenas una vaga idea de qué es realmente el dinero... no investiga la verdad ni busca la certeza. Todo lo que desea es información sobre posibilidades y comprensión de las posibilidades o riesgos que la situación inmediata determina

mundo de los contemporáneos en tanto realidad directamente vivenciada donde tienen lugar mis ejecuciones prácticas. Por otro lado, en Schütz el 'mundo de la vida' nunca es pensado como objetivo y estático, sino, por el contario, como totalidad dinámica.

$7 \quad$ Actitud natural presupone: la existencia corpórea de otras personas, que esos cuerpos están dotados de conciencias esencialmente similares a la mía, que las cosas del mundo exterior son similares para nosotros y tienen fundamentalmente el mismo sentido, que puedo entrar en acciones y relaciones recíprocas con mis semejantes, que puedo hacerme entender por ellos, que el mundo social y cultural está dado históricamente de antemano como marco de referencia y que la situación en la que me encuentro en todo momento es solo en pequeña medida creada exclusivamente por mí (Schütz y Luckmann (1973 [1977]: 26). 
para el resultado de sus acciones" (1964 [1974]:97-98).

En definitiva, en el mundo de la vida tiene lugar la experiencia cotidiana y la comprensión del sentido (significado); actuando en él y sobre él, los actores se hacen y hacen el mundo, convirtiéndolo en el entorno vital en el cual cabe comprender la relación con lo heredado, con los otros y con la naturaleza. Es el mundo experimentado inicialmente por los sujetos en la actitud natural, un mundo complejo e incoherente (con todos lo grados de nitidez $)^{8}$, él único que "puede constituirse en un mundo circundante, común y comunicativo... es, por consiguiente, la realidad fundamental y eminente del hombre" (Schütz y Luckmann 1973 [1977]: 25). Pese a todas las diferencias posibles de encontrar entre los distintos individuos y grupos sociales "el conocimiento de la vida cotidiana basta para entenderse con el prójimo, los objetos culturales y las instituciones sociales; en resumen, con la realidad social” (Schütz 1962 [2003]:76-77).

\subsection{El presente vivido y el ejecutar coti- diano como constitutivos del mundo de la vida cotidiana}

Podemos señalar que el 'mundo de la vida', en un sentido genérico, comprende la configuración de un 'plexo' que articula dimensiones, relaciones, actores, sentidos, modos de conocimiento, y que se extiende vinculando los pasados, presentes y futuros (Walsh 1932 [1993]). Además, constituye un mundo presupuesto y público en el sentido que su existencia y ordenamiento no dependen del actor, aconteciendo de manera organizada el acervo de experiencias e interpretaciones que han realizados nuestros antecesores, y que se presenta a nuestra subjetividad así como a la de nuestros contemporáneos (Nebenmenschen) y congéneres (Mitmenschen) para ser experimentado, interpretado y construido, y que se proyecta hacia otro tiempo para constituirse en el mundo dado para otros.
Sobre lo anterior, lo que interesa realzar a Schütz es la particularidad que adquiere el 'mundo de la vida' en la interacción social o mundo de la vida cotidiana (López 1995; Leal 2006), y conocer cómo esta predisposición institucionalizada de sentido que conforma el mundo, se hace presente y es dinamizada por el actor social concreto -interprete- en actitud natural (Salas 2006). Hablamos de cómo el 'mundo de la vida' cobra 'sentido de realidad' en la centralidad del actor social -desde una matriz social y un situación biográfica determinada-, en un 'plano' o 'dimensión' que se define en función de la disposición - a actuarde 'ego' en tiempo y espacio, éstas últimas, categorías referenciales a partir de las cuales se establecen relaciones de alteridad posibles conforme a la experiencia vital ${ }^{9}$. Es por ello que “... al hombre alerta le interesa principalmente el sector del mundo de su vida cotidiana que está a su alcance y que en el espacio y el tiempo se centra a su alrededor" (Schütz 1962 [2003]:209). Schütz supone que el 'mundo cotidiano' es un campo de acción o dominación en el cual llevamos a cabo nuestro proyecto. No hay un interés teórico, puesto que en 'actitud natural' gobierna “.... un interés eminentemente práctico, causado por la necesidad de satisfacer los requerimientos básicos de nuestra vida" (Schütz 1962 [2003]:213). Para plasmar nuestros objetivos, “... debemos dominar lo que está presente en ellos y transformarlos... no solo actuamos y operamos dentro del mundo de la vida, sino también sobre él, es una realidad que modificamos mediante nuestros actos y que, por otro lado, modifica nuestras acciones" (Schütz y Luckmann 1973 [1977]:28). En definitiva, dentro del 'mundo de la vida cotidiana', mundo en el cual tienen lugar las ejecuciones prácticas -ejecutar cotidiano-; el 'yo' actúa en tanto 'realidad eminente', realidad inmediata, la cual vivencio en 'actitud natural', pero sobre el cual tienen lugar los actos ejecutivos que permiten la relación con y la modificación del mundo (relación pragmática). Como señala Schütz, “... el mundo del ejecutar cotidiano es el arquetipo

\footnotetext{
$8 \quad$ Es complejo en su multiformidad. Además, no solo se compone de subjetividades, sino también de interpretaciones de esas subjetividades. Como especifica Schütz, "El sistema de conocimiento así adquirido -incoherente, incongruente y solo parcialmente claro- adquiere para los miembros del endogrupo la apariencia de una coherencia, claridad y congruencia suficientes como para ofrecer a cualquiera una probabilidad razonable de comprender y se comprendidos" (1964 [1974]:98).

$9 \quad$ Siguiendo la perspectiva de Schütz, se podría resaltar el apelativo de cotidiano para dar a entender precisamente lo que es simultaneo y común para el actor (diario vivir), es decir, nos situamos en un plano de contemporaneidad y de la materialidad desde el cual nos relacionamos.
} 
de nuestra experiencia de la realidad y todos los demás ámbitos de sentido pueden ser considerados como sus modificaciones" (Schütz 1962 [2003]:217).

Así consignado, en el 'mundo de la vida' podemos distinguir dominios que involucran diversos grados de relaciones entre actores sociales (efectivas y probables) y de estos con otros mundos, entre los cuales Schütz distingue el 'mundo social de los contemporáneos' (soziale Mitwelt), 'mundo de los predecesores' (Vorwelt) y 'mundo de los antecesores' (Folgewelt), los dos últimos mundos, alejados de mi experiencia vital y con los cuales me relaciono a través de la ideación.

\section{Figura $N^{\circ} 1$}

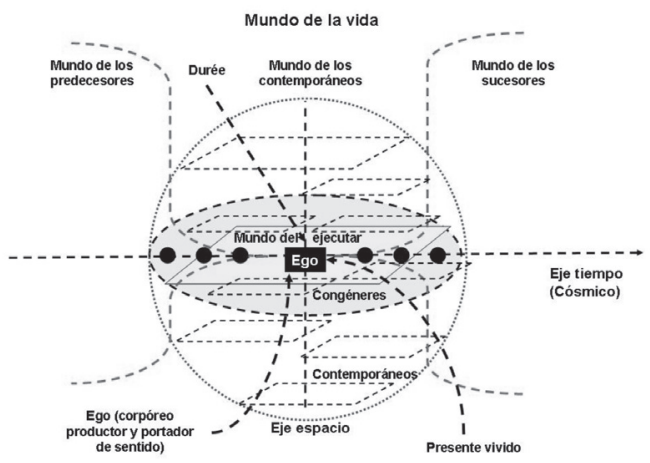

Como señala Schütz,

“... puedo también conocer un mundo social que existió antes que yo y que en ningún punto se recubre con parte alguna de mi propia vida. Con respecto a este dominio, el 'mundo social de los predecesores' (Vorwelt), o historia, solo puedo ser un observador y no un actor. Por último, sé que hay aún otro mundo, habitado también por otros, que existirá cuando yo ya no exista, un mundo social de los sucesores (Folgewelt), hombres de los cuales no sé nada como individuos y con cuyas vivencias no puedo tener contacto personal. De hecho, solo conozco sus vivencias típicas suponiendo que estas últimas serán las mismas que las de mis contemporáneos y las de mis predecesores. Este es un mundo que solo puedo captar vagamente pero nunca vivenciar en forma directa" (1932[1993]:173).
En el 'mundo de los contemporáneos' (soziale Mitwelt) coexiste y es simultáneo a mi duración, sin embargo no todas las relaciones posibles se constituyen por experiencia directa. Los 'congéneres' (Mitmenschen) corresponden a “... los otros yoes del mundo de la realidad social directamente vivenciada, y 'contemporáneos' (Nebenmenschen) a los otros yoes del mundo de los contemporáneos" (Schütz 1932[1993]:172). Para Schütz, esta distinción implica que vivencio mis 'congéneres' directamente y a su vivencia, en tanto que mis 'contemporáneos', aun cuando próximos, no son accesibles de manera directa, sino que se infieren sobre la base de evidencia directa de la vivencias típicas que debiesen tener. Es por ello que es posible afirmar que en el 'mundo de los contemporáneos' se pueden establecer varios tipos de relaciones.

Al respecto, Schütz plantea que en el 'mundo cotidiano' no todas las relaciones se experimentan 'cara a cara' o por relación directa o 'corpórea' entre un ‘yo' y un 'otro'. Es por ello que el 'mundo de la vida cotidiana' no es solo el mundo del presente directamente vivido o de las ejecuciones. La situación 'cara a cara' supone un presente vivido compartido, en la cual la relación nosotros-otros requiere la copresencia de los coparticipantes. Compartimos un ambiente en el cual, además, es posible aprehender las expresiones corporales del otro como suceso externo y como factor del proceso mismo de comunicación. Así, y solo así “... puede el copartícipe ver el sí-mismo de su semejante como una totalidad indivisa en un presente vivido"(Schütz 1962 [2003]:208). También existe un cuasi presente, en el cual se interpreta el resultado de la comunicación del otro sin participar en el proceso en curso del acto comunicativo, como es lo que procede de la lectura de una carta, un libro, etc. Es decir, siendo común un tiempo histórico, me vinculo con 'contemporáneos' con quienes nunca me encuentro. Por tanto, no todo en el mundo esta a nuestro 'alcance manipulatorio' o de actuación directa; sin embargo el acervo de experiencias anteriores permite la 'locomoción', la anticipación de que el objeto distante puede entrar en contacto con nosotros comprensivamente. Para Schütz, el 'mundo a su alcance' implica que el mundo de la vida vincula recíprocamente la contemporaneidad (mundo al alcance actual) con el pasado y el 
presente (mundo al alcance potencial). Distingue aquí un mundo al alcance del pasado recuperable, que refiere a lo que estuvo antes a mi alcance y puede recuperarse (la idealización que permite volver a hacer lo que hice), pudiendo mi área manipulatoria pasada seguir funcionando en mi presente como potencial. También, refiere un mundo al alcance futuro, el que no está ni ha estado nunca a mi alcance, pero al cual puedo acceder por idealización. De este modo, la totalidad del mundo social es un mundo dentro de mi alcance posible o asequible, y así,

"... incluye, no solo el mundo que está dentro del alcance actual del Otro, sino también los mundos que se hallan dentro de su alcance recuperable o asequible, y a la totalidad del sistema así entendido a todos los estratos diferentes del mundo social muestra todos los matices que se originan en las perspectivas de socialización, como la intimidad y la anonimia, la amenidad y la familiaridad, la proximidad y la distancias sociales, etc., que gobiernan mis relaciones con asociados, contemporáneos, predecesores y sucesores" (Schütz 1962 [2003]:212).

2 La comprensión (Verstehen): conformación del mundo social y subjetividad

"Debo comprender mi mundo de la vida en el grado necesario para poder actuar en él y poder operar sobre él" (Schütz y Luckmann 1973 [1977]:28).

\subsection{Definición y características de la com- prensión (Verstehen)}

Para Schütz, el sujeto social actúa en la realidad, por tanto tiene capacidad de recrearla. Esto se desprende del supuesto de que el 'mundo cotidiano' es “... el campo de acción y orientaciones posibles, organizado alrededor de su persona según el esquema específico de sus planes y las significatividades que derivan de ellos" (1967 [1974]:22). Esta capacidad radica en la comprensión (Verstehen), esa “... particular forma experiencial en que el pensamiento de sentido común toma conocimiento del mundo social cultural" (1962 [2003]:77), y se relaciona con el carácter significativo, dado y aprendido del mundo de la vida. Aquí el autor establece una diferencia respecto a propuestas elaboradas por otras tradiciones o tendencias en ciencias sociales ${ }^{10}$, para situarla en tanto condición constitutiva de la realidad y cualidad inherente a todo actor social (propia del ser) ${ }^{11}$. De este modo, la comprensión hace inteligible la realidad, y nos permite conocer y actuar en el mundo sociocultural.

La 'comprensión' tendría una base en la experiencia, ya que cualquier actor social aprende a través de diversas formas de socialización las significaciones que permiten entender al “... prójimo, los objetos culturales y las instituciones sociales (Schütz 1962 [2003]:28). Hace posible saber -o poder saber- por qué Pedro se persigna ante una iglesia, qué lleva a Julio a interesarse por la política partidista, qué implica un semáforo con la luz roja encendida, cual es la utilidad de un ordenador. Estas operaciones nos remiten a la 'comprensión', a la capacidad de hacer inteligible el mundo en el cual actuamos $-y$ que nos permite actuar en él-, en tanto provisto de motivos y fines, es decir, con sentido. La comprensión es la capacidad de presuposición de conocimiento actual y potencial del sentido de las acciones humanas y su producción, que deviene en actitud natural, ya que “... la experiencia de la existencia de otros seres humanos y del sentido de sus acciones es, sin duda, la primera y más original observación empírica que hace el hombre" (1962 [2003]:78). No actuamos en un mundo ajeno o extraño, sino en un mundo cercano y comprensible -no continuamente cuestionado- en base a esquemas que me permiten vivenciarlo a la manera de lo típico ${ }^{12}$. Como señalan Schütz y Luckmann:

10 V. gr., para Weber, Verstehen corresponde a un método que define la aproximación de una ciencia particular, la ciencia social.

11 Tras esta conceptualización se desprenden, ineludiblemente, problemas epistemológicos relativos al cómo comprender los motivos de la acción de otro, y metodológicos que conciernen a cómo elaborar conceptualizaciones objetivas de estructuras subjetivas de sentido.

12 Lo típico y lo único refieren a posibilidades de vivenciar o significar una acción, evento, objeto, etc. Como señala Schütz “... el hecho que yo haga una cosa u otra, y también qué rasgos de un objeto o sucesos dados consideraré como individualmente únicos y cuáles como típicos, dependerá de mi interés actual y del correspondiente sistema de significatividades; en resumen, del problema práctico o teórico que tenga 'a mano'” (1962 [2003]:80). 
"Cada paso de mi explicitación y comprensión del mundo se basa, en todo momento, en un acervo de experiencia previa, tanto de mis propias experiencias inmediatas como de las experiencias que me transmiten mis semejantes, y sobre todos mis padres, maestros, etc. Todas estas experiencias, comunicadas e inmediatas, están incluidas en una cierta unidad que tienen la forma de mi acervo de conocimiento, el cual me sirve como esquema de referencia para dar el paso concreto de mi explicitación del mundo. Todas mis experiencias en el mundo de la vida se relacionan con ese esquema, de modo que los objetos y sucesos del mundo de la vida se me presentan desde el comienzo en su carácter típico; en general, se me aparecen como montañas, piedras, árboles y animales, y más específicamente, como serranías, como robles, aves, peces, etc." (1973 [1977]:28).

En tanto capacidad de los actores sociales, la comprensión tendría las siguientes características:

- Es una cualidad humana.

- Es una creación sociocultural, producida por medio de la socialización (es estructurada y permite reciprocidad de perspectivas, es genética o de origen social, es distributiva y por tanto parcializada).

- Es el resultado de un proceso de aprendizaje o aculturación.

- Es pública (no privativa de un observador) debido al carácter intersubjetivo de la acción, y por tanto controlable por otro (verificabilidad intersubjetiva).

- Permite predicciones (basada en acciones y acontecimientos típicos).

- Es subjetiva, en tanto permite acceder al sentido que la acción tiene para un actor en circunstancias biográficamente determinadas, en contraste con un coparticipe o un observador indirecto. Es decir, “... el actor, y solo él, sabe lo que hace, por qué lo hace, cuándo y dónde comienza y termina su acción" (Schütz 1962 [2003]:80).

- Es tipificada, en tanto comprendo acciones de acuerdo a esquemas típicos de motivos y fines, de actitudes y personalidades en los cuales una conducta resulta un ejemplo, y a su vez, pasan a ser motivos de mis propias acciones.
- Es diversa -no unívoca -, pues remite al punto de vista del actor social, es decir, a su situación y posición en el mundo de la vida (Lebenswelt); mi "situación biográficamente determinada". En este sentido, puede concordar o discordar con la comprensión lograda por otro u otros actores sociales.

\subsection{Conformación del mundo social y subje- tividad en la comprensión (Verstehen)}

En cierta medida, la 'comprensión' es constitutiva del 'mundo de la vida' en tanto lo hace posible, 'le da forma' y coherencia, ya que el mundo no es experimentado como caótico e inconexo porque es comprendido. Esto tiene que ver con la dimensión ontológica de la comprensión -con esa capacidad propia del ser humano-, pero también con la dimensión epistemológica -se liga el comprender con el conocer-. La comprensión tiene un soporte en el conocimiento (herencia social, que se transmite por la socialización); yo comprendo por que conozco - me es familiar o típico-, la realidad así me es inteligible. En la comprensión del mundo yo y los otros vivimos (posibilita la comunicación); formamos parte de él, actuamos en él y contribuimos a su reproducción. Por ello, la comprensión es indisociable de la actuación; mi actitud natural en el mundo reproduce el entorno vital -en la relación con otros-, por ende, lo conforma a la manera de lo típico.

La interacción social se desarrolla en el 'mundo de la vida', nutriéndose de las interpretaciones que nos llevan a comprender el mundo y a los otros en el transcurrir de las vidas. Pero es central en la conceptualización desarrollada por Schütz la idea de que el 'mundo de la vida' es pre reflexivo, es decir, presupuesto. Su estructuración pasa desapercibida para el actor que ejecuta la acción, no siendo reflexionada y constituyéndose en un sustento incuestionado (pero cuestionable) donde se asienta lo común y lo típico. Esto quiere decir que el actor social actúa en el mundo y lo interpreta por medio de tipificaciones mediante las cuales reconoce en el mundo social otros semejantes (más próximos o más anónimos). Estas tipificaciones conforman lo que podríamos llamar estilos de operar familiares y reconocibles para los actores 
sociales que comparten una matriz social de conocimientos y experiencias (Salas 2006), y que, por ende, transfieren una competencia interpretativa elemental que otorga la posibilidad de comprender a las rutinas, motivos y causas de otros. Como el actor experiencia de manera directa su mundo, puede corroborar por medio de la 'reciprocidad de perspectivas' que sus 'contemporáneos' experimentan ese mundo de forma semejante a la suya, es decir, "Independientemente de la aceptación de que no estamos solos en este mundo, existe el axioma elemental de la posición 'natural' de que otros hombres viven y experimentan el mundo como nosotros [tesis de la intercambiabilidad], o en todo caso, bajo circunstancias parecidas [cláusula ceteris paribus]" (Luckmann 1992 [1996]:40). En palabras de Schütz,

"Nuestra relación con el mundo social se basa en la hipótesis de que, a pesar de todas las variaciones individuales, nuestros semejantes experimentan los mismos objetos de una manera sustancialmente similar a nosotros, y viceversa, y también que nuestro esquema de interpretación y el de ellos muestran la misma estructura típica de significatividades. Si se desploma esta creencia en la identidad sustancial de la experiencia intersubjetiva del mundo, queda anulada la posibilidad misma de establecer la comunicación con nuestros semejantes" (Schütz 1964 [1974]:139).

Por ello, en tanto funcionen los esquemas significativos de referencia como formas legitimadas por nosotros y nuestros 'congéneres' en el 'mundo de la vida', o sea, con sentido para nuestras experiencias así como para los resultados de nuestras acciones, este se reproduce sin contradicción o cuestionamientos.

Nacemos en un mundo compartido (intersubjetivo y público), lo cual no impide que podamos desarrollar experiencias particulares y únicas; es decir, frente al marco sociocultural heredado que define la comprensión ${ }^{13}$, coexiste la variabilidad que aporta la vivencia subjetiva del actor. Esto se debe a que, como se ha señalado en otros pasajes, la interpretación del mundo no es igual para todos, y por ende no todos los actores se relacionan de la misma manera ni orientan por los mismos motivos y fines. Aquí juega un papel fundamental la 'experiencia biográfica', desde la cual el actor social hace suyo el mundo, trasladando las experiencias desde el anonimato a su mismidad o historia de vida - desde círculos más directos a aquellos indirectos-. Mi biografía, por tanto, se compone del conjunto de experiencias en la que se combinan diversos elementos según grados de proximidad y exclusividad que alcancen mis relaciones, haciendo de ese fragmento de mi experiencia algo único (Salas 2006).

Así, la comprensión del 'mundo de la vida cotidiana' llevada a cabo por un actor social se encuentra mediada por su biografía (subjetividad), desde la cual vislumbran tanto las posibilidades como las limitantes referidas a lo que pueden o no hacer en el mundo, lo que se afecta por el orden de prioridades respecto de las cuales fija su atención en relación con lo que resulta central. En este sentido, la 'comprensión' se puede tornar diversa y matizada en el mundo social (dependiendo de las esferas de realidad en que participe), debido a que mi aquí y ahora constituyen las coordenadas en la cuales mis acciones se estructuran en significados que le asigno a los fenómenos, conformando la base a partir de la cual mi comprensión se resitúa en tanto intersección de tiempo cósmico y el espacio concreto con el acontecer de ‘ego' y su tiempo interior o ‘durée'.

\section{Subjetividad, intersubjetividad y tempo-} ralidad en la acción social

"El actor situado dentro del mundo social... lo experimenta principalmente como un campo de sus actos actuales y posibles, y solo de forma secundaria como objeto de su pensamiento. En la medida en que le interesa el conocimiento de su mundo social, organiza este conocimiento, no en términos de un sistema científico, sino de la significatividad para sus acciones" (Schütz 1964 [1974]:96).

\subsection{Acción, subjetividad e intersubjetividad}

Schütz hereda de Weber el concepto de acción y el postulado referido a la interpreta-

13 Marco en el cual el actor ocupa una posición social definida por su estatus y rol dentro del sistema social, además de una posición moral e ideológica. 
ción subjetiva, y profundiza en él situándolo en el 'mundo de la vida cotidiana' y ligándolo a la percepción y a la relevancia de las realidades experimentadas por el sujeto. El punto de atención de Schütz esta dado en la compresión de la acción social en tanto sentido del cual todo actor dota a sus acciones (López 1995). En esta línea, es fundamental la incorporación que Schütz efectúa de la tesis de la 'tensiones de conciencia' propuesta por Bergson, esto, en tanto permite diferenciar la acción social de otras manifestaciones humanas que tienen lugar en la vida cotidiana, poniendo de este modo acento en la asignación de sentido que 'ego' - ejecutante de la acción - imprime. Bergson sostiene que la vida consciente muestra un número indefinido de planos que van desde el sueño hasta la acción, implicando cada uno de ellos una tensión específica. La tensión mayor se visualiza en el plano de la acción, donde también se sitúa el mayor interés en el hacer frente a la realidad y satisfacer sus requisitos; la menor tensión y falta total de interés se ubica en el plano de la ensoñación. El principio regulador de la vida conciente se denomina 'atención a la vida' (attention à la vie), y es a partir de la cual se definen una serie de operaciones fundamentales para la vida cotidiana que permiten articular la corriente de pensamiento en un flujo continuo ${ }^{14}$.

En tanto situable en el mayor plano de tensión, Schütz define acción (Handeln) como,

“... el proceso por el cual un actor, en la vida cotidiana, determina su conducta futura después de haber considerado varios cursos de acción posibles. Con el término acción designaremos la conducta humana como proceso en curso que es ideada por el actor de antemano, es decir, que se basa en un proyecto preconcebido" (1962 [2003]:86).
Se diferencia del comportamiento en la medida que éste refiere a experiencias que están provistas de sentido desde la subjetividad y que emanan de nuestra vida espontánea sin necesidad de intención (plano de menor tensión); es decir, la 'acción' comprende un comportamiento ideado de antemano con base en un proyecto preconcebido. Por otro lado, se diferencia del acto (Handlung), ya que este designa el resultado del proceso en curso, es decir, la acción cumplida. Como hemos manifestado, toda acción implica una decisión previa que puede ser tan básica como es el hecho de decidir entre actuar y no hacerlo (Salas 2006) ${ }^{15}$. En este sentido, la acción puede ser 'manifiesta' o 'latente', esto, si se tiene o no la intención de cumplir el proyecto o crear el estado de cosa proyectado. La 'acción latente' puede estar dotada o no de propósito, pasando de la ensoñación o fantasía a acción cuando subiste el interés por llevarla a efecto. Las 'manifiestas', en cambio, se insertan en el mundo exterior mediante movimientos corporales, sin importar si están acompañadas de la intención de realizarla, ya que toda acción manifiesta es una ejecución ${ }^{16}$. De este modo, la acción siempre constituye un ejercicio consciente, y en tanto esté situada en el plano del ejecutar (acción manifiestas), representará la mayor tensión de la conciencia -estado de alerta - en el marco de la denominada 'atención a la vida' (attention à la vie). Al respecto, Schütz señala que el 'estado de alerta'

“... delinea todo ámbito pragmáticamente significativo del mundo. Esta significatividad, a su vez, determina la forma y el contenido de nuestra vida de conciencia... la tensión de nuestros recuerdos y, con ellos, el dominio de nuestras remembranzas de las vivencias del pasado, determinando al mismo tiempo el dominio de nuestras expectativas. Determina el contenido porque todas nuestra vivencias actuales son modi-

14 Estas son: la determinación de la función y alcance de nuestra memoria, hace vivir nuestras experiencias presentes 0 volvernos en actitud reflexiva hacia experiencias pasadas en busca de significado.

15 Como señala Schütz, “...puedo provocar un estado futuro de cosas no interviniendo. Tal abstención proyectada de la acción puede ser considerada en sí misma como una acción” (1932 [1993]:86). Lo anterior cobra sentido, ya que el no actuar como decisión de no actuar puede tener una consecuencia sobre el mundo. V. gr. En una reunión sindical, un asociado puede decidir no dar su opinión sobre las vulneraciones laborales sufridas a manos del empleador, debido a que está presente alguien que estima es cercano a la gerencia. Por consiguiente, no se constata tal vulneración, y la situación no es tematizada por la dirección sindical.

16 La ejecución es “... una acción en el mundo externo basada en un proyecto y caracterizada por la intención del producir el estado de cosas proyectado mediante movimientos corporales” (Schütz 1962 [2003]:201). 
ficadas a través de proyectos anteriormente esbozados y su realización" (Schütz y Luckmann 1973 [2001]:45).

Como señalamos, el interés principal de Schütz es el sentido subjetivo que el actor asigna a la acción y cómo se establecen las relaciones intersubjetivas (López 1995; Leal 2007). En esta línea, la acción social es interpretada como tipificación desde la cual se visualiza la conducta propia como la de los otros, siempre entendida como provista de sentido y propósito. Se encuentra dotada de sentido al consignar que otro actor posee las mismas cualidades que yo (tan humanos como los míos), y por tanto son provista de sentido para ambos, como para todos los copartícipes. Así, el actor social, en el marco de su situación biográficamente determinada, define el sentido y situación de la cual forma parte, estructurando la realidad y actuando en el 'mundo de la vida'. La 'situación biográfica' actúa como la instancia de vida desde la cual elaboro tipificaciones afincadas en la información prevista; es lo que Schütz denomina la disponibilidad o reserva de conocimiento a mano (stock of knowledge at hand), y es a partir de lo cual el mundo adquiere cierta familiaridad, orientando las acciones y decisiones con naturalidad. Esta tipificación permite proyectar los actos teniendo en consideración las regularidades depositadas en mis experiencias - presupuestos de la experiencia-, las que hacen posible la acción social. La permanencia de las tipificaciones obedece a propiedades de idealización que son parte del proceso ' $y$ así-sucesivamente' (und-so-weiter), en tanto que la disponibilidad de conocimiento a mano obtenido de los semejantes y formado en la experiencia mantiene validez 'hasta nuevo aviso'. Así, el esquema tipificado se regula mediante el 'siempre-puedo-volver-a-hacerlo' (Ich kann-immer-wieder), habría que agregar, de manera similar.

Otro aspecto importante de consignar guarda relación con definir los 'motivos' que están tras las acciones, lo que resulta clave tanto para la comprensión de la perspectiva temporal en la cual se inserta el proyecto, como también de la acción social. Schütz establece una diferenciación entre los 'motivos para' (Un-zu-motive) y los 'motivos porque' (Weil-Motive).
Los primeros, 'motivos para', se relacionan directamente con la interpretación subjetiva del sentido -conciencia del actory con el dominio del futuro. Pueden ser definidos como las razones que llevan al actor a emprender acciones para alcanzar un objetivo en el futuro, remitiéndose su existencia al hecho de su realización (una especie de sistema de planificación). Es subjetivo al referir la experiencia del actor que vive en el curso de su actividad, y significa lo que este tiene realmente en vista y que da sentido final a su acción. Según Luckmann (1992 [1996]), la encadenación de 'motivos para' está claramente prescrita en la perspectiva temporal, es decir, en un presente dirigido hacia un futuro, siendo este futuro efectivo en tanto representación actual del futuro. Su emergencia remite retrospectivamente al pasado, a una prehistoria que forma parte componente de la historia global de la vida del agente; "Abarca sobre todo las experiencias de actos correspondientes almacenadas en el bagaje cognoscitivo que originalmente se hallan a la base de las estructuras de relevancia subjetiva -igualmente solidificadas en la historia de la vida- (Luckmann p.59). Los 'motivo-para' constituirían un acto proyectado o estado de cosas imaginado y que debe producirse a través de la acción, en tanto implican objetivos y fines que se procuran lograr (Salas 2006).

Los segundos, 'motivos-porque', corresponden al dominio del pasado, y podrían entenderse como predisposiciones ligadas a los antecedentes biográficos y del contexto donde el actor define su situación y desde los cuales justifica la conducta actual (una especie de sistema de personalidad que reúne experiencias previas, actitudes, hábitos, etc.). Schütz señala que "Estas experiencias lo han llevado a actuar como lo hizo. Lo que está motivado, en una acción del tipo porque, es el proyecto de la acción misma" (1962 [2003]:88). Para un observador, estos poseen un carácter objetivo, ya que deben ser reconstruidos a partir del acto realizado o estado de cosas creado en el mundo externo por parte del actor. Para Luckmann (1992 [1996]), los auténticos 'motivos porque' pueden retrotraerse tan lejos como se quiera, sin que se llegue propiamente a un fin, dándonos en algún momento por satisfechos en función del marco de nuestro interés; "Siempre que deseemos interrumpir, 
la dirección temporal se dirigirá al pasado. El proyecto en gestación del futuro proviene de este pasado" (Luckmann p.60).

En definitiva, la comprensión de los actos de otros pasa por conocer las motivaciones 'para' y 'porque'. Sin embargo, la posibilidad de captar el detalle de las motivaciones (todas sus implicaciones) trasciende nuestra capacidad presencial; por ello se recurre a las tipificaciones en tanto motivos, finalidades, situaciones y medios típicos. Así, el conocimiento de los 'otros' posee distintas intensidades en tanto son 'próximos íntimos' o 'lejanos anónimos', siendo la tipificación disponible la que permite familiaridad respecto de quien conozco como de quien nunca he visto (puedo hacer referencia a actorestipos que se desenvuelven en situaciones típicas). El aquí y ahora definido por la centralidad del actor (ego) es la base desde la cual se establece proximidades que van desde la relación 'cara a cara' hasta el total anonimato. En este marco, Schütz entiende que la relación social es una 'conexión intersubjetiva de motivos' (Luckmann (1992 [1996]:26), en tanto que puedo anticipar que los 'motivos para' de mi actuación constituyen 'motivos porque' de la reacción del 'otro' y viceversa. Como ya se ha señalado, lo que confirma que el 'mundo de la vida' está provisto de sentido, se relaciona con la constatación del que el 'otro' me responde, y además que suponemos la existencia de motivaciones 'para' y 'porque' similares entre 'ego' y 'álter'. Esta posibilidad de comunicación redunda en la existencia de un esquema de referencia que es común para nuestros actos; en definitiva, el sentido que el mundo tiene para otros actores lo tiene para mí. Esta es la 'reciprocidad' e 'intercambiabilidad de perspectivas', que en tanto hace sentido en mi experiencia, me lleva a la interrogación acerca de los motivos de 'otros' como base para el entendimiento de la interacción social. En resumen, la interpretación de la subjetividad del 'otro' procede a través de su aprehensión como tipo en el marco del contexto de interacción en el cual tiene lugar una situación también típica. A partir de ello se constituye un sistema de motivos que deriva de las relaciones sociales recíprocas, sistema que se basa en las tipificaciones en tanto ordenación jerárquica de motivos.

\subsection{Intersubjetividad y alteridades en la temporalidad de la acción social}

Como se especificó en el primer apartado, lo relevante para Schütz es el 'mundo de la vida' en tanto realidad eminente, mundo al alcance del actor en el cual ejecuta las acciones y pone en marcha su proyecto; es decir, el mundo al alcance efectivo (Reichweite), accesible a mi experiencia inmediata. Esto remite a la primacía de la contemporaneidad, esto es, del aquí y ahora del actor, y como desde estas coordenadas vivencia el mundo y se relaciona con los 'contemporáneos'. Sin embargo, Schütz no desconoce la relevancia de las realidades ajenas a la experiencia directa del actor social, sino más bien plantea que se organizan cognoscitivamente en la simultaneidad de la vida de ese. Al respecto señala que,

“... el sí-mismo alerta integra en su ejecutar, y por su intermedio, su presente, pasado y futuro en una dimensión temporal específica; se concreta como totalidad en sus actos ejecutivos, y por medio de ellos se comunica con Otros y organiza las diferentes perspectivas espaciales del mundo de la vida cotidiana" (1962 [2003]:201).

Es por ello que la acción social se encuentra completamente inmersa en la temporalidad, es decir, implica decisiones que enlazan el pasado, el presente y el futuro. Sin embargo, el actor social elabora dicha síntesis, no en el plano del tiempo cósmico o histórico, sino por medio de la internalización vivencial de este tiempo, dando lugar a una dimensión personal o interior del tiempo denominada 'durée', En este sentido, la simultaneidad de la acción ejecutiva obedece a sucesos del mundo exterior e interior, en tanto flujo unificado que da origen al 'presente vivido'. El 'presente vivido' responde a la intersección de la 'durée' y el tiempo cósmico, y es la dimensión en la cual transcurre mi flujo de conciencia, así como la instancia en la cual comparto subjetividad con otros actores sociales. Como señala Natanson (1962 [2003]), la intersubjetividad transcurre en el 'presente vivido' en el cual nos comunicamos. En tanto simultaneidad, se transforma en la esencia de la intersubjetividad ya que implica la captación de la subjetividad del 'álter-ego' en el instante que vivo mi 'flujo de concien- 
cia'; es esta captación simultánea y recíproca lo que hace posible nuestro ser conjunto en el mundo.

La 'durée', expresión que Schütz recoge de Bergson, se constituye en concepto relevante en tanto pone en relación la dimensión recuperable y anticipable de la acción, y por ende, hace posible, a través de esta efectuación, la relación del pasado y futuro con el presente. A esta forma particular de presente, Schütz la denominó 'presente especioso', siendo en este que las acciones y vivencias traen consigo el pasado y el futuro, entrelazando el tiempo cósmico (de la naturaleza inanimada) y el estándar o cívico (social) que también se impone como realidad objetiva (construida por las convención social, y que Schütz lo grafica en la frase 'envejecemos juntos'). En lo que respecta a la acción, la 'durée' hace patente la recuperación, otorgando la posibilidad de traer al presente actuaciones pasadas (con base en la sedimentación de experiencias), es decir, trasladando el pasado a la vivencia actual. Por otro lado, permite acciones potenciales proyectadas hacia el futuro, poniendo en un 'área manipulatoria' próxima aspectos que no han sido asequibles de manera directa, haciendo posible la anticipación, siempre dependiente del acervo de conocimiento y experiencias.

En términos de la acción social, esto puede ser entendido de la siguiente forma. La acción social constituye un flujo continuo en el cual no cabe la posibilidad de tener en vista las experiencias propias de mi actuación en curso en 'modo presenti'. Con actitud reflexiva puedo captar mi acto efectuado o las fases iniciales de este como actuación pasada o pretérita perfecta. Pero también puede referirme en 'modo futuri exacti' debido a que toda acción es un proyecto, es decir, está dotada de propósito ${ }^{17}$-implicando meditación y planificación-. En este sentido, se exige reflexión acerca del su curso efectivo para alcanzar un resultado -en estado de alerta-, esto, a través de determinaciones tales como escoger, mediante juicios evaluativos, el mejor curso de acción. Tal reflexión corresponde a un experimento o proyección mediante la cual se busca efectuar combinaciones diversas de elementos seleccionados de los hábitos e impulsos para discernir cómo sería la acción resultante si se emprendiera (Schütz 1932 [1993]), y cuya planificación demanda esbozar cada paso de mi acción futura de manera de lograr el objetivo fijado, visualizando el estado de cosas que será producido por mi acción. La anticipación recae no sobre la acción, sino sobre el acto que provocará aquel estado de cosas proyectado; antes de su realización, debo situarme en el futuro perfecto, en el cual la acción tomó curso y fue concretada, de manera de reconstruir los pasos aislados de la acción que provocaron los resultados esperados. Su fundamentación se encuentra en actos realizados con anterioridad, de los cuales ya poseo una experiencia.

Por otro lado, las coordenadas 'espaciotiempo' (respecto de ego) permiten establecer relaciones de alteridad que dan lugar a tipos de interacción en planos diversos, esto, porque las formas en que se sitúa mi experiencia en la realidad es variada. Por ello, “... experimento a los hombres en diversas perspectivas, y mi relación con ellos se ordena según variados niveles de proximidad, profanidad y anonimia en la vivencia" (Schütz y Luckmann 1973 [2001]:76). De esta manera, el 'otro' puede ser referido como mi predecesor (de quien puedo llegar a saber por información indirecta e imprecisa), mi sucesor (a quien no llegaré a conocer), mis contemporáneos (con quienes cohabito pero experimento de manera indirecta) y mis 'asociados' o ‘congéneres' (con quienes establezco relaciones directas). Esta clasificación da lugar a distintos tipos de relaciones posibles, entre las que se pueden reconocer la relación nosotros y la relación ellos.

La relación nosotros transcurre en el mundo circundante (Umwelt), es decir, en el plano en que el 'otro' comparte conmigo un sector del espacio y tiempo del mundo de la vida, plano que hace posible el 'encuentro' cara-cara (el 'otro' como corporeidad viva). Lo anterior da lugar a la 'orientación Tú' (DuEinstellung), que deriva de experimentar al

17 En definitiva, lo fundamental en la acción es la dotación de intencionalidad o propósito; en tanto que no todas las proyecciones implican un propósito, todo proyecto que solo se desarrollan en la mente sin existir intención de concreción, constituye un mero proyecto y no una acción. Por ende, para comprender la acción social, se debe tomar conocimiento de la proyección de la acción y los motivos que la definen. 
'otro' en persona como un igual a 'mí', captándolo en su peculiaridad (un hombre en particular o un tipo de particular de hombre) en un proceso de aprehensión y tipificación concreta. La 'orientación Tú' se diferencia según la atención que el 'otro' presta a mi existencia; la 'orientación Tú' unilateral (yo atiendo al 'otro', pero para ese 'otro' paso inadvertido) y 'orientación Tú' recíproca (mutua atención). Esta última da lugar a una relación social que Schütz denomina 'relación Nosotros', en la cual

"Él y yo, nosotros, compartimos mientras dura el proceso un presente vivido común, nuestro presente vívido, que le permite y me permite decir 'Nosotros experimentamos este suceso juntos'... ambos, -él, que se dirige a mí, y yo, que lo escucho- estamos viviendo en nuestro presente vívido mutuo, dirigidos hacia el pensamiento que debe ser realizado en el proceso de comunicación y por él. Envejecemos juntos" (1962 [2003]:207).

Así, la 'relación Nosotros' queda definida por un alto grado de intimidad e inmediatez, en tanto existe familiaridad biográfica de un 'otro', y compromete el flujo de vivencias personales de los actores partícipes en la interacción, teniendo lugar el fenómeno de la intersubjetividad. La relación 'pura' se manifiesta cuando los coparticipantes son conscientes de ellos mismos y participan asociadamente en la vida de cada uno por un tiempo, por breve que sea. Por su parte, la inmediatez permite acceder a la conciencia del 'otro' en tanto se tienen 'a la mano' manifestaciones de su experiencia subjetiva. Por otro lado, se hace posible someter a revisión las tipificaciones que poseemos de ese 'otro', y eventualmente modificarlas. En efecto, en la 'relación Nosotros' el actor parte desde ciertos supuestos típicos que actúan como anticipación de lo que los 'otros' son y piensan; sin embargo, en la interacción tiene lugar la modificación constante de las acciones y de las concepciones de los 'otros'. Podemos considerar que las motivaciones del 'otro' son del mismo orden que las mías (poseemos un proyecto común o similar), en tanto que otras veces, en la rela- ción, es posible constatar que no existe congruencia, y que por tanto ese 'otro' no es un cercano o un asociado. La simultaneidad de la interrelación hace de esto algo de sumo complejo; en tanto es imposible conocer con seguridad al 'otro', la relación se trasforma en un proceso en que se ponen a prueba diversos planes y recetas que van y vienen (relación iterativa). Como por lo general no existe una reflexión en el curso de la acción, son estas recetas las que cobran relevancia, las que pueden ser abandonadas cuando emergen problemas ${ }^{18}$, modificando el curso de la acción y dirigiéndose, previa reflexión, hacia respuestas apropiadas.

Cuando se producen alteraciones en nuestro flujo de conciencia, la atención es atraída hacia otros ámbitos de sentido, teniendo lugar la disminución del 'estado de alerta', dirigiendo el 'acento de realidad' a otros sectores del 'mundo de la vida'. Lo que queda fuera de foco, puede asumir la forma de componentes constitutivos del estilo cognoscitivo direccionado al nuevo ámbito en el que comenzamos a incursionar, perfilando su propio 'acento de realidad', es decir, el sector del mundo que se vuelve relevante. En esta tensión, un actor definido como un 'congénere' o 'asociado' puede quedar fuera del foco de nuestro interés, constituyéndose en un 'contemporáneo' con quien sostengo 'relaciones Ellos'. Lo anterior se ejemplifica en la siguiente cita de Schütz

"Cuando tengo una evocación de ti, por ejemplo, te recuerdo como eras en la relación-nosotros concreta conmigo, como una persona única en una situación concreta... Te recuerdo como una persona vívidamente presente para mí con un máximo de síntomas de vida interior, como alguien cuyas vivencias he presenciado en el proceso real de su formación, a quien yo, durante un tiempo, iba conociendo cada vez mejor, cuya vida consciente fluía en una sola corriente junto con la mía y cuya conciencia estaba cambiando continuamente de contenido. Sin embargo, ahora que estás fuera de mi experiencia directa, no eres más que mi contemporáneo, alguien que meramen-

Schütz denomina conmoción a la modificación en la tensión de nuestra conciencia como efecto de una alteración en la 'atención a la vida', lo que trae consigo un cambio en la forma de vivenciar la realidad. 
te habita el mismo planeta que yo. Ya no estoy en contacto con el tú viviente, sino con el tú de ayer. Tú, en verdad, no has cesado de ser un yo viviente, pero tienes ahora un nuevo yo; y aunque soy un contemporáneo de él, mi contacto vital con él se ha interrumpido. Desde el último momento en que estuvimos juntos, has tenido nuevas vivencias y las has enfocado desde nuevos puntos de vista. Con cada cambio de vivencia y enfoque te has transformado en una persona levemente distinta. Pero en cierto modo yo omito tener presente esto en la praxis de mi vida diaria. Llevo tu imagen conmigo, y sigue siendo la misma. Pero entonces oigo decir, quizá, que tú has cambiado. Y luego comienzo a mirarte como a un contemporáneo; no cualquier contemporáneo, sin duda, sino alguien a quien conocí en un tiempo íntimamente" (1932 [1993]: 206-207).

Así, un 'asociado' o ‘congénere' puede trasformarse en un contemporáneo, como también un 'contemporáneo' puede trasformarse en un 'asociado'. En tanto la ubicación espacial cambia, se hace posible establecer relaciones 'cara-cara' (proximidad) o relaciones indirectas (lejanía), existiendo por tanto una transición gradual y continua que puede situar al 'otro' en distintos planos y niveles de relación.

El tipo de relaciones que establezco con los 'contemporáneos' se denomina 'relaciones Ellos'. En estas, el contacto 'cara-cara' no es posible, por lo cual median relaciones más o menos anónimas e indirectas con actores tipo, conductas tipo o cursos de acto tipo (personal o funcionario), lo que implica la imposibilidad de someter a escrutinio el desenvolvimiento del 'otro' en la interacción. Como señalan Schütz y Luckmann, "Mientras que en el encuentro social experimento así concretamente a un semejante de manera inmediata... capo en su existencia fáctica y el ser de tal y cual modo de un contemporáneo únicamente por medio de tipificaciones derivadas" (Schütz y Luckmann 1973 [2001]:87). De este modo, la 'orientación Ellos' (Ihr-Einstellung) se infiere desde mi conocimiento y del mundo en general (actores situados en el entramado social como tipos de actores: policías, empleados de correos, etc.) ubicándose en un contexto de sentido objetivo, siendo posible además trazar relaciones tipificadas a partir de inferencias extraídas de las experiencias directas anteriores de semejantes (experiencias pasadas) o por comentarios que me transmite otro acerca de un tercero. Al consistir las relaciones sociales entre 'contemporáneo' en ser captado como un 'otro', estas adquieren un carácter probable y anticipatorio, en el sentido de que espero que el 'contemporáneo' hacia el cual me oriento se oriente hacia mí con una tipificación adecuada y complementaria.

Como señalan Schütz y Luckmann, "Las relaciones entre contemporáneos se determinan mediante las probabilidades subjetivas de la complementariedad de sentido de la tipificación usada por los copartícipes" (Schütz y Luckmann 1973 [2001]:97). Aquí intervienen actos conscientes que presumimos, orientan la conducta del copartícipe, y que presentan diversos grados de uniformidad. Schütz y Luckmann señalan que a mayor uniformidad de una esquema de tipificación, mayor es la probabilidad subjetiva (de la mera conjetura hasta la certidumbre subjetiva) de confirmación de expectativas de cada copartícipe. Los esquemas de tipificación pueden estar uniformados en el marco de instituciones (en cuanto al ámbito de uso y tipo), tradicionalmente determinados (el ámbito de uso y tipo de uso tienen una distribución social general), o referir al esquema de uniformación que concierne a tipos de curso de actos coordinados con un esquema racional medio-fines. Lo anterior es relevante, pues mis experiencias pueden cambiar las tipificaciones que dirigen mi 'orientación Ellos' con limitaciones radicales; esto solo puede suceder en medida muy limitada mientras no se modifique la esfera de interés que definió el uso original del tipo. Si bien en la 'relación Nosotros' puedo hallar siempre confirmación de la congruencia de mis experiencias respecto de las de mi 'álter', en la 'relación Ellos', este tipo de confirmaciones no se hace posible, lo que remite a formas indirectas como introducir o más bien recuperar tipificaciones de quienes fueron semejantes para ponerlas en uso.

\section{Comentario final}

Como señala Natanson (1962 [2003]), sería erróneo sostener que la obra de Schütz es solo una extensión del pensamiento de 
Husserl o una mera aplicación del método fenomenológico a las ciencias sociales. Schütz recupera la vida cotidiana como objeto de las ciencias sociales (Reynoso 1998), ahondando en el sentido social que tienen las relaciones en el mundo de la vida, incorporando la dimensión histórico contextual de la biografía, en tanto determinante en la estructuración de tipificaciones, acciones típicas y recetas del sujeto (Salas 2006). Demostró que la fenomenología es una vía plausible para realizar el estudio comprensivo de la realidad social y de las relaciones entre los sujetos, precisando los presupuestos, estructura y significación del mundo de la vida experimentado intersubjetivamente en actitud natural, haciendo patente la riqueza conceptual y la aplicabilidad empírica de su programa (López 1995).

A diferencia de la solución filosófica que Husserl da al problema de la intersubjetividad -trascendental-, Schütz introduce la dimensión socializadora. El sujeto es un ser social, y por ende, la construcción de la conciencia no proviene de un exclusivo ensimismamiento inmanentismo, sino de la interacción en la cual nos hacemos al compartir con otros ciertas ideas y conocimientos (Leal 2007). En este sentido, no hablamos de los 'otros' como representaciones de la conciencia -meros objetos-, ya que al reconocer que también son poseedores de conciencia, pueden interactuar y desenvolverse tal como yo en el mundo de la vida. Este salto representa, en filosofía, el abandono de la conciencia "monádica", en la línea de lo que plantea Leibniz; hasta Schütz, la mayoría de las perspectivas referían a individuos como entidades autocontenidas, es decir, se ponían límites o se cuestionaba la posibilidad de conocer o representar lo que los sujetos piensan, o mejor dicho, su intencionalidad o motivación para la acción. De este modo se soluciona el dilema epistemológico que implicaba el conocer en profundidad qué fundamenta la acción social intersubjetiva y se supera la idea cartesiana de que somos cuerpos que van por el mundo portando una mente (Schuster 1995).

Para el conocimiento profundo del mundo de la vida, Schütz retoma a Husserl, reconociendo que cada sujeto social posee una perspectiva singular desde la cual ve el mundo, esto quiere decir que la conciencia solo es referencial -refiere a contenidos u objetos-, por lo cual para dar cuenta del conjunto se requiere de la totalidad de las perspectivas de los sujetos sociales - la suma de escorzos en Husserl-. Esta tarea es la del científico social; recurriendo a las tipificaciones -que son propias de la vida cotidiana - se logra sintetizar características particulares a manera de superar la individualización o nominalización. Por otro lado, se debe rescatar el esfuerzo que hace Schütz por clarificar la idea de que la realidad es interpretada y que, más allá de la existencia de leyes sociales que nos condicionan, es la interpretación y la puesta en escena de la intencionalidad del actor -su capacidad comprensiva - fundamental para el abordaje de lo 'sociocultural' (Schuster 1995). La dimensión empírica es la que hace posible tener acceso a la comprensión de los otros, es decir, a los fundamentos de su accionar por medio de la observación, de la conversación, de la contextualización etc. Yo puedo comprender porque en cierto modo comparto lo que el otro realiza o señala.

Por tanto, podríamos decir que tenemos acceso comprensivo al mundo en la interacción con otros sujetos - somos parte del mundo que habitamos- que han sido socializados en el curso de la vida, adquiriendo conocimientos similares. Sin embargo, el problema se suscita si quiero conocer e interactuar en otros contextos culturales con los cuales no tengo la continuidad de las pautas de socialización. Al respecto, Schuster (1995) $)^{19}$ señala que esta es una gran limitante en la perspectiva comprensiva de Schütz, y uno de los puntos sobre los cuales surgen los mayores cuestionamientos, sobre todo de la antropología (Reynoso 1998). Para Schuster (1995), el trabajo de Schütz se situó en el marco de la sociología y no de disciplinas como la antropología, obviando problemáticas ligadas al relativismo cultural y a las posibilidades de acceso comprensivo de otras culturas.

Aun cuando en el 'El forastero. Ensayos de psicología social’ (1964 [1974]) esboza al-

Este argumento también fue planteado en el seminario "Debates contemporáneos en epistemología de las ciencia sociales”, dictado por Federico Schuster en la Universidad Nacional de La Plata, Argentina, entre abril y junio de 2010. 
gunas consideraciones generales al respecto, hay que reconocer que el acceso comprensivo a mundos diferentes, fuera de una tradición cultural concreta, es un aspecto no desarrollo por Schütz. Sin embargo, esto no quiere decir que desde la antropología no se puedan rescatar ciertos desarrollos; de hecho los ha incorporado con fuerza a nivel epistemológico y metodológico a través de los estilos fenomenológicos y comprensivistas desarrollados en antropología por Geertz (1973 [2003]) ${ }^{20}$ y Agar (1992), entre muchos otros. Resulta interesante para la época la conjunción que propone entre 'Lebenswelt', 'realidades múltiples', 'acento de realidad' y 'ámbitos finitos de sentido', proponiendo problemáticas que la antropología incorporó a partir de los años 70 a partir del profundo cuestionamiento a la concepción monolítica, autocontenida y esencialista de cultura que la visualizaban como un todo homogéneo (Wolf 1982 [1987]; Friedman (1994 [2001]; Appadurai (1996 [2001]). Problematizar en torno a la 'diversidad intracultural', es decir, a la existencia de realidades múltiples - ancladas en la biografía de los sujetos- en el marco de un mismo grupo social resulta muy interesante para relativizar el manejo que se efectúa de este concepto en el ámbito especializado y político. Además, permite plantear interrogantes acerca de la profundidad con la cual algunos cientistas sociales se han hecho cargo del concepto de interculturalidad, sin resolver primero qué es 'lo cultural' y cómo se recoge el problema de las múltiples realidades presentes en un mismo grupo.

Más allá de las críticas ${ }^{21}$, el trabajo de Schütz continúa siendo estimulante para disciplinas como la antropología, ciencia que se ha definido como aquella capaz de dar cuenta de la otredad, más allá de la incorporación sistemática que ha adquirido en sociología. Nuevas lecturas son sin siempre estimulantes para emprender reflexiones respecto al método etnográfico y su puesta en marcha, así como para el desarrollo de estrategias y técnicas más allá de los aportes de la etnometodología, la etnografía reflexiva y fenomenológica (Hammersley y Atkinson 1983 [1994]; Agar, 1992; Woods 1996 [1997]; Ghasarian 2002 [2008]) y la teoría fundamentada (Strauss \& Corbin 1998 [2002]). En este sentido, Schütz abre caminos para reflexionar acerca del oficio del investigador social.

\section{Bibliografía}

AGAR, M. (1992). Hacia un lenguaje etnográfico. En Carlos Reynoso (ed.), El surgimiento de la antropología postmoderna (pp.117-137). Barcelona: Gedisa.

ALEXANDER, J. (1987 [1992]). Las teorías sociológicas desde la segunda guerra mundial. Análisis multidimensional. Barcelona: Gedisa.

APPADURAI, A. (1996 [2001]). La modernidad desbordada. Dimensiones culturales de la globalización. México: Fondo de Cultura Económica.

BENGOA RUIZ DE AZÚA, J. (1992). De Heidegger a Habermas. Hermenéutica y fundamentación última en la filosofía contemporánea. Barcelona: Herder.

FRIEDMAN, J. (1994[2001]). Identidad cultural y proceso global. Buenos Aires: Amorrortu.

GHASARIAN, C. (2002 [2008]). "Por los caminos de la etnografía reflexiva". En GHASARIAN, C., ABÉLĖS, M., BELLIER, I., et al. De la etnografía a la antropología reflexiva. Buenos Aires: Ediciones Sol.

GIBERT, J.(1999). Los conceptos de 'mundo de la vida' y 'mundo cotidiano' en la obra de Schütz. Revista Colombiana de Sociología (4), 1, 54-61. URL: http: //repositorio.uvm.cl/ $\mathrm{gsdl} / \mathrm{collect} / \mathrm{sociol} / \mathrm{index} / \mathrm{assoc} / \mathrm{HASH} 01 \mathrm{~d} 7$. $\mathrm{dir} / \mathrm{doc}$. pdf [20-2-2010].

GIDDENS, A. (1967 [1993]). Las nuevas reglas del método sociológico. Buenos Aires: Amorrortu.

$20 \quad$ Incluso Geertz (1973 [2003]:302) recupera las categorías de relacionales de alteridad propuestas por Schütz aplicándolas al análisis de los esquemas culturales balineses.

21 No es la intención profundizar en las múltiples críticas realizadas a la propuesta de Schütz. Sin embargo, podemos mencionar: la denuncia del psicologismo, la confusión en el uso de categorías fenomenológicas, el problema de la consideración de la experiencia como amontonamiento de partes, la imposibilidad de una síntesis entre fenomenología y sociología, la necesidad de visualizar la negociación de los significados desde puntos de vista diferenciales - no totalmente libres-, la insuficiencia del postulado de adecuación, etc. (Giddens 1967 [1993]; Alexander 1987 [1992]; Reynoso 1998). 
HAMMERSLEY, M. y ATKINSON, P. (1983 [1994]). Etnografía. Métodos de investigación. Barcelona: Paidós.

LEAL, R. (2006). La sociología interpretativa de Alfred Schütz. Reflexiones en torno a un planteamiento epistemológico cualitativo. Revista Alpha, 23, 201-213. URL: http://www.scielo. cl/scielo.php?pid=S0718-22012007000200015\&script=sci_arttext

LEAL, R. (2007). Aportes teórico-metodológicos de la fenomenología al desarrollo cualitativo de las ciencias sociales en Alfred Schütz. Revista Alpha, 25, 215-225. URL: http://www.scielo. cl/scielo.php?pid=S0718-22012007000200015escript=sci_arttext

LÓPEZ SÁENZ, M. C. (1995). La sociofenomenología de Alfred Schütz: entre el constructivismo y el realismo. Revista Paper, 47, 55-74. URL: ddd.uab.cat/pub/papers/02102862n47p55. pdf [20-2-2010].

LUCKMANN, T. (1996). Teoría de la acción social. Barcelona: Paidós.

MILONE, R. (2007). Lebenswelt husserliana y concepción semántica de las teorías. Ideas y Valores. Revista colombiana de filosofía, Universidad Nacional de Colombia, 135, 89-99.

NOVELLA, J. (1998). Crisis de las ciencias, Lebenswelt y teoría crítica. Revista de Filosofía, Universidad de Murcia, 16, 103-118.

REYNOSO, C. (1998). Corrientes antropológicas contemporáneas. Buenos Aires: Biblos.
SALAS, R. (2006). El Mundo de la vida y la fenomenología sociológica de Schütz. Apuntes para una filosofía de la experiencia. Revista de Filosofía, 15, 167-199.

SCHUSTER, F. (1995). Exposición, hermenéutica y ciencias sociales. En A.A. V.V. El oficio del Investigador. Rosario: Homo sapiens.

SCHÜTZ, A. (1932 [1993]). La construcción significativa del mundo social. Introducción a la sociología comprensiva. Barcelona: Paidós.

(1962 [2003]). El problema de la realidad social. Buenos Aires: Amorrortu.

(1964 [1974]). Estudios sobre teoría social. Buenos Aires: Amorrortu.

SCHÜTZ, A. y LUCKMANN, T. (1973 [1977] [2001]). Las estructuras del mundo social. Buenos Aires: Amorrortu.

STRAUSS, A. y CORBIN, J. (1998 [2002]). Bases de la investigación cualitativa. Técnicas y procedimientos para desarrollar la teoría fundamentada. Medellín: Editorial Universidad de Antioquia.

WOLF, E. (1982 [1987]). Europa y la gente sin historia. México: Fondo de Cultura Económica.

WOODS, P. (1996 [1997]). Investigar el arte de enseñar. El uso de la etnografía en la educación. Barcelona: Paidós. 